\title{
Experimental nuclear level densities and interpretation within the microcanonical ensemble
}

\author{
M. Guttormsen ${ }^{a}$, U. Agvaanluvsan ${ }^{b}$, R. Chankova ${ }^{a}$, A.C. Larsen ${ }^{a}$, J. Rekstad ${ }^{a}$, \\ A. Schiller ${ }^{c}$, S. Siem ${ }^{a}$, N.U.H. Syed ${ }^{a}$, and A. Voinov ${ }^{d}$ \\ ${ }^{a}$ Department of Physics, University of Oslo \\ P.O.Box 1048 Blindern, N-0316 Oslo, Norway \\ ${ }^{b}$ Lawrence Livermore National Laboratory \\ L-414, 7000 East Avenue, Livermore, California 94551, USA \\ ${ }^{c}$ National Superconducting Cyclotron Laboratory \\ Michigan State University, East Lansing, Michigan 48824, USA \\ ${ }^{d}$ Department of Physics and Astronomy \\ Ohio University, Athens, $\mathrm{OH} 45701$, USA \\ E-mail: magne.guttormsen@fys.uio.no
}

\begin{abstract}
Statistical properties of atomic nuclei are studied as function of the number of neutrons. The experimental method is based on particle- $\gamma$ coincidences measured in light-ion reactions with one charged ejectile. From particle- $\gamma$ coincidences, level densities and radiative strength functions can be simultaneously extracted from one and the same data set. Mid-shell nuclei show significant odd-even entropy differences interpreted as the single-particle entropy of the valence nucleon. However, these properties change when approaching closed shells. A model within the framework of the microcanonical ensemble is presented and compared with data from the molybdenum isotopes. The odd-even entropy difference disappears in the vicinity of the $N=50$ shell, and an overshoot of positive parity states is predicted.
\end{abstract}

International Symposium on Nuclear Astrophysics - Nuclei in the Cosmos - IX

25-30 June 2006

CERN 

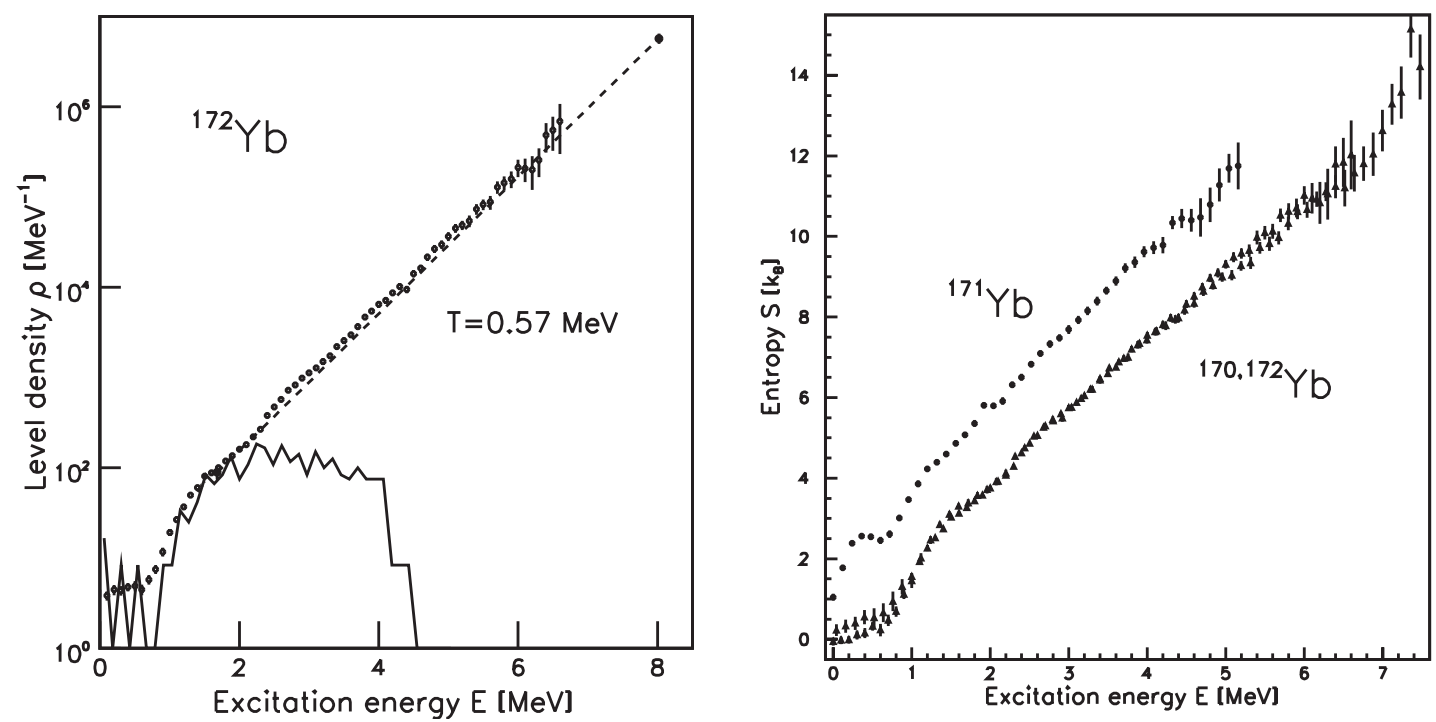

Figure 1: Experimental data based on the $\left({ }^{3} \mathrm{He}, \alpha\right)$ reaction on ${ }^{171,172,173} \mathrm{Yb}$ targets. Left panel: experimental total level density (filled circles) and constant temperature extrapolation (dashed line) to the level density based on neutron resonance data at the neutron binding energy $B_{n}$. The jagged line is based on counting known discrete levels. Right panel: entropies deduced for ${ }^{170,171,172} \mathrm{Yb}$.

\section{Introduction}

Atomic nuclei at low excitation energy are characterized by the motion of pairs of nucleons, known as Cooper pairs, moving in time reversed orbitals. With increasing excitation energy, the description becomes more complicated as Cooper pairs are broken by collective (Coriolis force) or intrinsic (temperature) excitations.

In order to investigate the breaking of Cooper pairs, experiments were conducted at the Oslo Cyclotron Laboratory (OCL) using a 38 to $45 \mathrm{MeV}^{3} \mathrm{He}$ beam on self-supporting targets. Particle- $\gamma$ coincidences were detected with an array of 28 collimated $\mathrm{NaI} \gamma$-ray detectors and eight Si particle telescopes placed at $45^{\circ}$ with respect to the beam. The investigated reactions are $\left({ }^{3} \mathrm{He},{ }^{3} \mathrm{He} \gamma\right)$ and $\left({ }^{3} \mathrm{He}, \alpha \gamma\right)$ from the 0-n channel. By applying the Brink-Axel hypothesis, the level density can be extracted from the primary $\gamma$-ray spectra, which are measured at all initial excitation energies up to the neutron binding energy. Details on the experimental set-up and data analysis are given elsewhere [1].

Figure 1 shows typical level densities $\rho$ for well deformed rare-earth nuclei as function of excitation energy $E$. The left panel also shows data based on counting known levels and neutron resonance spacing data. In the right panel the level density is transformed into entropy by $S(E)=$ $\ln \rho(E) / \rho_{0}$, where $\rho_{0}=2.2 \mathrm{MeV}^{-1}$. It is striking that the $S$ curves are almost identical for ${ }^{170,172} \mathrm{Yb}$. The odd ${ }^{171} \mathrm{Yb}$ system reveals an increase of $\Delta S=1.5-2.0$, which is interpreted as the entropy carried by the valence particle (or hole) outside the heated even-even core [2].

This simple picture of spectators becomes less valid when going to lighter nuclei, where few single-particle orbitals are available for the valence nucleon. The strongest effect appears when a shell is closed. Here, more configurations can only be produced by crossing the shell gap. The most dramatic reduction is found for the double magic ${ }^{208} \mathrm{~Pb}$ nucleus, which has about 100 times 
fewer levels than the lighter ${ }^{202} \mathrm{Hg}$.

Recently [3, 4], a unique set of level densities and radiative strength functions in the ${ }^{93-98} \mathrm{Mo}$ isotopes was reported. The data clearly show the evolution of level density when going from one neutron $\left({ }^{93} \mathrm{Mo}\right)$ to six neutrons $\left({ }^{98} \mathrm{Mo}\right)$ outside the $N=50$ shell gap. The aim of the present work is to describe this deformation dependence within a transparent model of quasiparticles.

\section{Model}

The model is described within the microcanonical ensemble, where the excitation energy $E$ is well defined. The single-particle energies $e_{\mathrm{sp}}$ are taken from the Nilsson model for an axially deformed core described by the quadrupole deformation parameter $\varepsilon_{2}$. Furthermore, the model depends on the spin-orbit and centrifugal parameters $\kappa$ and $\mu$ and the oscillator quantum energy $\hbar \omega_{0}$ between the harmonic oscillator shells. Each single-particle orbital is characterized by the spin projection $\Omega$ on the symmetry axis.

Within the BCS model, the single-quasiparticle energies are defined by

$$
e(\Omega)_{\mathrm{qp}}=\sqrt{\left[e(\Omega)_{\mathrm{sp}}-\lambda\right]^{2}+\Delta^{2}},
$$

where the Fermi surface $\lambda$ is adjusted to reproduce the number of particles in the system and $\Delta$ is the pairing gap parameter. The quasiparticle energy due to proton $(\pi)$ and neutron $(v)$ excitations is given by

$$
E_{\mathrm{qp}}\left(\Omega_{\pi}, \Omega_{v}\right)=\sum_{\left\{\Omega_{\pi}^{\prime} \Omega_{v}^{\prime}\right\}}\left[e_{\mathrm{qp}}\left(\Omega_{\pi}^{\prime}\right)+e_{\mathrm{qp}}\left(\Omega_{v}^{\prime}\right)+V\left(\Omega_{\pi}^{\prime}, \Omega_{v}^{\prime}\right)\right] .
$$

A residual interaction $V$ is defined between the aligned $\left|\Omega_{\pi}+\Omega_{v}\right|$ and anti-aligned $\left|\Omega_{\pi}-\Omega_{v}\right|$ configurations as a Gaussian distribution centered at zero energy with a width of $50 \mathrm{keV}$. Collective energy terms are schematically added by

$$
E=E_{\mathrm{qp}}\left(\Omega_{\pi}, \Omega_{v}\right)+A_{\mathrm{rot}} R(R+1)+\hbar \omega_{\mathrm{vib}} v,
$$

where $A_{\text {rot }}$ is the rotational parameter and $R=0,2,4, \ldots$ is the rotational quantum number. The vibrational motion is described by the phonon number $v=0,1,2, \ldots$ and the oscillator quantum energy $\hbar \omega_{\text {vib }}$.

The set of proton and neutron orbitals $\left\{\Omega_{\pi}^{\prime} \Omega_{v}^{\prime}\right\}$ is picked out by using a random generator. Technically, we repeat this process until all possible energies $E_{\mathrm{qp}}\left(\Omega_{\pi}, \Omega_{v}\right)$ are visited. The advantage of the present model is a fast algorithm that may include several protons and neutrons in a large space of single-particle states.

\section{Discussion}

The experimental [3] level densities of ${ }^{93-98}$ Mo are shown in the left panel of Fig. 2. The ${ }^{93,94}$ Mo nuclei display very similar level densities, indicating that the odd-even entropy difference has vanished, in contradiction to the case of ytterbium (see Fig. 1). The scattered neutrons are presumably filling mainly the orbitals $d_{5 / 2}$ and $g_{7 / 2}$ above the $N=50$ shell gap, which should limit the available single-particle orbitals. Indeed, the ${ }^{93} \mathrm{Mo}$ isotope has about ten times fewer levels 

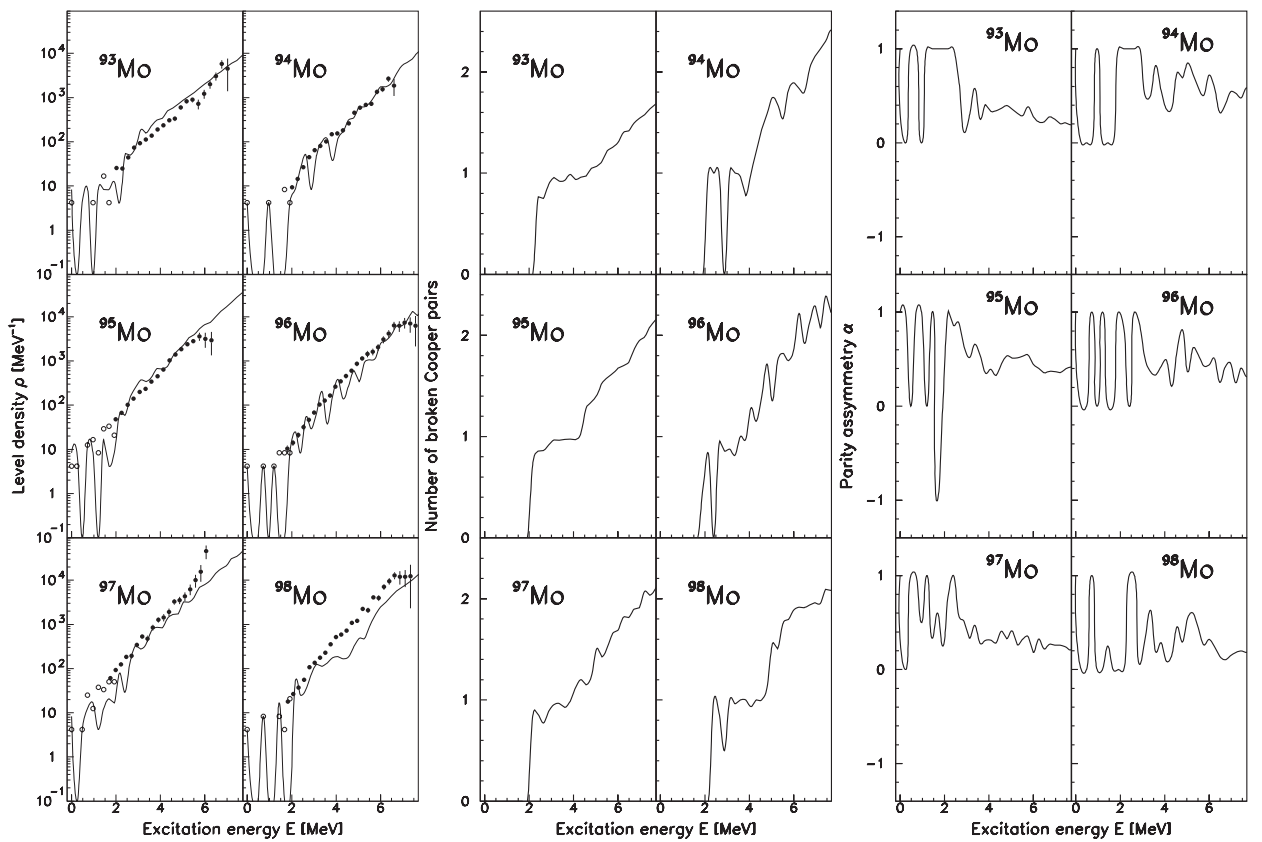

Figure 2: Experimental and simulated level densities for molybdenum isotopes (left panel). The next two panels show the simulated number of broken Cooper pairs and parity asymmetry $\alpha$.

than predicted from global level-density formulas. The protons are not expected to be responsible for the reduced level density, since there are no significant energy gaps between orbitals below or above the proton Fermi surface.

In the calculation we have adopted standard Nilsson parameters $\kappa=0.06$ and $\mu=0.32$ with oscillator quantum energy of $\hbar \omega_{0}=41 A^{-\frac{1}{2}} \mathrm{MeV}$. The deformation parameter $\varepsilon_{2}$ is taken from known $B(E 2)$-values [5]. The rotational and vibrational terms contribute only significantly to the total level density in the lower excitation region. Therefore, $A_{\text {rot }}$ and $\hbar \omega_{\text {vib }}$ are determined from the first and second excited states in the even-even molybdenums, respectively. For the odd-mass nuclei, these values are interpolated in order to obtain reasonable values. The adopted pairing gap parameters $\Delta_{\pi}$ and $\Delta_{v}$ are taken from the five-mass formula of Ref. [6].

The experimental [3] and calculated level densities are shown in the left panel of Fig. 2. In view of the fact that no parameters have been tuned to obtain best agreement, the comparison is very satisfactory. The odd-even staggering and the decrease in density when approaching the $N=50$ shell gap are well reproduced. We see that the adopted values give nice agreements for the breaking of the first Cooper pairs in even-even nuclei as well as reproducing the log slope of the level densities for all isotopes.

The middle panel of Fig. 2 shows the theoretical number of Cooper pairs broken as function of excitation energy. The first pair breaks up at $E \sim 2 \mathrm{MeV}$ for all molybdenums. Which of the proton or neutron pairs that break at the lowest energy, depends on the pairing parameters and details of the single-particle schemes around the respective Fermi surfaces. The four quasiparticle regime starts around $4-5 \mathrm{MeV}$ and is most clearly seen for ${ }^{98} \mathrm{Mo}$; it may also be present in ${ }^{95} \mathrm{Mo}$. The pronounced plateau of one broken pair between 2.2 and $4.5 \mathrm{MeV}$ of excitation energy in ${ }^{98} \mathrm{Mo}$ 
could be responsible for the underestimate of the level density above $3 \mathrm{MeV}$. However, the higher experimental level density could also be due to coexistent nuclear shapes of this nucleus, signalized by a very low-lying second $0^{+}$state at $0.735 \mathrm{MeV}$. Such a second potential minimum could very well produce twice the number of levels and is not included in our calculations.

The present model also provides information on the parity asymmetry parameter given by [7]

$$
\alpha=\frac{\rho_{+}-\rho_{-}}{\rho_{+}+\rho_{-}} .
$$

Here, $\alpha$ gives -1 and +1 for pure negative and positive parity regions, respectively, and 0 when both parities are equally represented. The asymmetry parameter is easily transformed to the fraction of positive and negative parity states by $f_{ \pm}=(1 \pm \alpha) / 2$.

For molybdenum $(Z=42)$, the proton Fermi surface is situated in between the $g_{9 / 2}$ particle and $p_{1 / 2}, f_{5 / 2}$ and $p_{3 / 2}$ hole states, producing about the same number of positive and negative parity states. However, the neutrons favor positive parities since they occupy the $d_{5 / 2}$ and $g_{7 / 2}$ particle and $g_{9 / 2}$ hole states. In the right panel of Fig. 2 is shown the parity asymmetry $\alpha$ as function of excitation energy. All six isotopes show that the positive parity states dominate. Typically, around $2 \mathrm{MeV}$ of excitation energy we find almost only positive parity states with $\alpha \sim 1$. The asymmetry then drops with excitation energy, giving $\alpha=0.2-0.4$ at $8 \mathrm{MeV}$.

\section{Conclusion}

We have demonstrated that the concept of single-particle entropy works nicely for mid-shell nuclei. When approaching closed shells, the valence nucleons may have no single-particle orbitals available to create entropy. This fact reduces the overall entropy as well as the odd-even entropy difference. In addition, as shown for the molybdenums, a parity asymmetry occurs, which is expected to modify the relative intensities for $E 1$ and $M 1$ radiation in continuum.

Financial support from the Norwegian Research Council (NFR) is gratefully acknowledged.

\section{References}

[1] A. Schiller et al., Nucl. Instrum. Methods Phys. Res. A 447, 498 (2000).

[2] U. Agvaanluvsan et al., Phys. Rev. C70, 054611 (2004).

[3] R. Chankova et al., Phys. Rev. C 73, 034311 (2006).

[4] M. Guttormsen et al., Phys. Rev. C 71, 044307 (2005).

[5] T. Belgya et al. Handbook for calculations of nuclear reaction data: Reference Input Parameter Library. Available online at http://www-nds.iaea.org/RIPL-2/, IAEA, Vienna, 2005.

[6] A. Bohr and B.R. Mottelson, Nuclear structure, Vol. 1 (Benjamin, New York, 1969) 169.

[7] U. Agvaanluvsan, G.E. Mitchell, J.F. Shriner, Jr., and M. Pato, Phys. Rev. C 67, 064608 (2003). 